\title{
Aerodynamic Characteristics Calculation for New Generation Space Vehicle in Rarefied Gas Flow
}

\author{
Yuri Ivanovich Khlopkov, Vladimir Alekseevich Zharov, Zay Yar Myo Myint ${ }^{*}$, Anton Yurievich \\ Khlopkov
}

\begin{abstract}
Department of Aeromechanic and Flight Engineering, Moscow Institute of Physics and Technology, Zhukovsky, Russia Corresponding Author: zayyarmyomyint@gmail.com
\end{abstract}

Copyright $(2013$ Horizon Research Publishing All rights reserved.

\begin{abstract}
This paper deals with the computational analysis of aerodynamics of space vehicle entering the Earth's atmosphere (from the free-molecular regime to the continuum flow regime) in rarefied gas flow. The use of approximate engineering methods is the most favorable approach to solve problems of spacecraft aerodynamics at the stage of conceptual design. In this paper present the calculation results of aerodynamic characteristics of new generation space vehicle in free molecular, the transitional and continuum regimes by using engineering method. This method may useful to calculate aerodynamic characteristics of new generation space vehicle designs.
\end{abstract}

Keywords Space Technology, Hypersonic Vehicles, Aerodynamic Characteristics in Transitional Regime, DSMC, Rarefied Gas Dynamic, Engineering Method

\section{Introduction}

For developing and designing space vehicles, accurate prediction and evaluation of hypersonic aerodynamic and aerothermodynamic characteristics in the whole range of flow regimes, i.e., from the free-molecular regime to the continuum flow regime are greatly important. During de-orbiting, the spacecraft passes through the free molecular, then through the transitional regime and the finalized flight is in the continuum flow.

For the reentry flight in the upper atmosphere, where it is necessary to take into account the molecular structure of a gas, kinematics models are applied, in particular, the Boltzmann equation and corresponding numerical methods of simulation. In the extreme case of free-molecular flow, the integral of collisions in the Boltzmann equation becomes zero, and its general solution is a boundary function of distribution, which remains constant along the paths of particles [1]. While aircraft are moving in a low atmosphere, the problems are reduced to the problems that can be solved in the frame of continuum theory or, to be more precise, by application of the Navier-Stokes equations and Euler equations. It is natural to create engineering methods, justified by cumulative data of experimental, theoretical and numerical results, enabling the prediction of aerodynamics and aerothermodynamic characteristics of complex bodies in the transitional regime [2]. The analysis of the aerodynamics and aerothermodynamic characteristics of hypersonic vehicle at high-altitude requires numerous numerical calculations $[3,4,5]$. To correctly simulate hypersonic flows, the flows must be understood and modeled correctly and this more true than in the numerical simulation of hypersonic flows.

Hypersonic must be dominated by an increased understanding of fluid mechanics reality and an appreciation between reality and the modeling of that reality [6]. The benefits of numerical simulation for flight vehicle designs are enormous: much improved aerodynamic shape definition and optimization, provision of accurate and reliable aerodynamic data and highly accurate determination of thermal and mechanical load [7]. Multi-parametric calculations can be performed only by using an approximation engineering approach. Computer modeling allows to quickly analysis the aerodynamic characteristics of hypersonic vehicles by using theoretical and experimental research in aerodynamics of hypersonic flows. The basic quantitative tool for study of hypersonic rarefied flows is direct simulation Monte Carlo method (DSMC) [8]. DSMC method is required large amount of computer memory and performance and unreasonable expensive at the initial stage of spacecraft design and trajectory analysis. The solution for this problem is the approximate engineering methods. The Monte Carlo method remains the most reliable approach, together with the local engineering methods, that provides good results for the global aerodynamic coefficients. The early work of [2] indicated that local engineering methods could have significant effect on aerodynamic characteristics of various vehicles.

The purpose of this work is to calculate aerodynamic characteristics of new generation space vehicle with the use of approximate engineering method. The method is suitable to calculate with taking into account the influence of Reynolds number, and provide good results for various 
vehicle shapes.

\section{Calculation Method}

Engineering based aerodynamic analyses were unreasonable expensive at the initial stage of space vehicle design. Difficulties of solving aerodynamic problems of over flow on bodies of rarefied gas caused the development of engineering semi-empirical methods based on the accumulated experimental data. When modeling the natural conditions necessary to consider the basic similarity criteria. The hypersonic aerodynamic coefficients most commonly used parameters are:

Mach number $\mathrm{M}$

$$
\mathrm{M}_{\infty}=\frac{\mathrm{V}_{\infty}}{a_{\infty}}
$$

The similarity parameter that governs these different flow regimes is the Knudsen number $\mathrm{Kn}$, defined as (rarefaction parameter):

$$
\mathrm{Kn}=\frac{\lambda_{\infty}}{L_{r e f}}=1.25 \sqrt{\gamma} \frac{\mathrm{M}_{\infty}}{\operatorname{Re}_{\infty}}
$$

Reynolds number Re

$$
\mathrm{Re}_{\infty}=\frac{\rho_{\infty} \mathrm{V}_{\infty} L_{r e f}}{\mu_{\infty}}
$$

where, $\lambda_{\infty}$ - the mean free path, $L_{\text {ref }}$ - reference length of vehicle, $\mu_{\infty}$ - the viscosity coefficient. In the hypersonic stabilization more efficiently used as of rarefaction criteria is not the Knudsen Kn number, as well as Reynolds number Re.

The Newtonian sine squared law indicated that the force varies as the square of the sine of the deflection angle. More than half century later indicated that Newton's sine-squared law was not very accurate and, indeed, the preponderance of fluid dynamic experience up to the present day confirms this finding, the exception to this is the modern world of hypersonic aerodynamics $[9,10,11,12]$. The introduction of modified Newtonian theory continues to be used often to generate approximation for the pressure acting on configurations in hypersonic flows $[13,14,15]$.

In this work we use the expressions for the elementary pressure forces and friction forces are applied in the form described in $[2,16,17]$.

$$
\begin{gathered}
p=p_{0} \sin ^{2} \theta+p_{1} \sin \theta \\
\tau=\tau_{0} \sin \theta \cos \theta
\end{gathered}
$$

here, coefficients $p_{0}, p_{1}, \tau_{0}$ (coefficients of the flow regime) are dependent on the Reynolds number $\operatorname{Re}_{0}=\rho_{\infty} \mathrm{V}_{\infty} L / \mu_{0}$, in which the viscosity coefficient $\mu_{0}$ is calculated at stagnation temperature $T_{0}$. Except Reynolds number the most important parameter is the temperature factor $t_{w}=T_{w} / T_{0}$, where $T_{0}, T_{w}$ are the stagnation temperature and surface temperature respectively.

The dependency of the coefficients of the regime in the hypersonic case must ensure the transition to the free-molecular values at $\operatorname{Re}_{0} \rightarrow 0$, and to the values corresponding to the Newton theory, methods of thin tangent wedges and cones, at $\operatorname{Re}_{0} \rightarrow \infty$. On the basis of the analysis of computational and experimental data, the empirical formulas are proposed

$$
\begin{gathered}
p_{0}=p_{\infty}+\left[p_{\infty}\left(2-\alpha_{n}\right)-p_{\infty}\right] p_{1} / z, \\
p_{1}=z \exp \left[-\left(0.125+0.078 t_{w}\right) \operatorname{Re}_{0 \text { eff }}\right], \\
\tau_{0}=3.7 \sqrt{2}\left[R+6.88 \exp \left(0.0072 R-0.000016 R^{2}\right)\right]^{-1 / 2}
\end{gathered}
$$

Here,

$$
\begin{gathered}
z=\left(\frac{\pi(\chi-1)}{\chi} t_{w}\right)^{1 / 2} \\
R=\operatorname{Re}_{0}\left(\frac{3}{4} t_{w}+\frac{1}{4}\right)^{-0.67} \\
\operatorname{Re}_{0 \text { eff }}=10^{-m} \operatorname{Re}_{0}, m=1.8(1-h)^{3}
\end{gathered}
$$

here, $h$ is a relative lateral dimension of the apparatus, which is equal to the ratio of its height to length.

The method proved to be good for the calculation of hypersonic flow of convex, not very thin, and spatial bodies. The local engineering method can be applied with the bodies are close to being spherical, and not applied in the case of very thin bodies [2].

A comparison of the experimental and calculated results (dashed curve) of drag coefficient for the sphere by using local engineering method $\left(\mathrm{M}_{\infty}=5.15-10, T_{w} / T_{0}=1\right)$ is performed in fig. 1 [2]. It can be seen that the influence is small. In figure 2 presented the results of drag coefficients for space shuttle with the use of this method [2].

Thus, the calculation of aerodynamic characteristics of the bodies by local engineering method in the hypersonic flow of rarefied gas in the transitional regime gives good result for drag coefficient for a wide range of bodies, and a qualitatively right result for lift coefficient. In this case, it is necessary to involve more complete models that take into account the presence of the boundary layer [2].

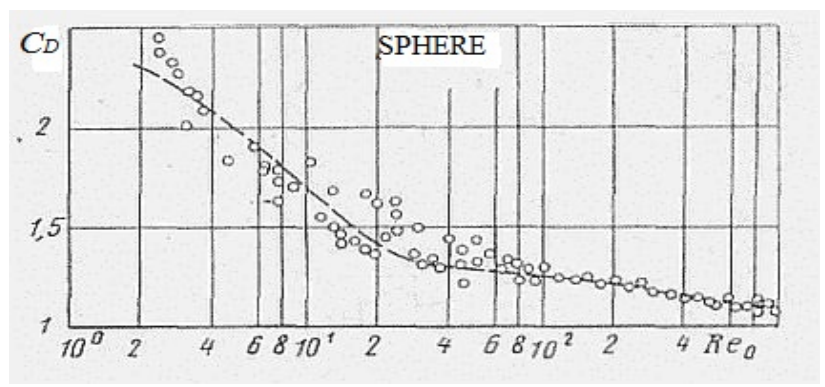

Figure 1. Drag coefficient vs $\mathrm{Re}_{0}$ 


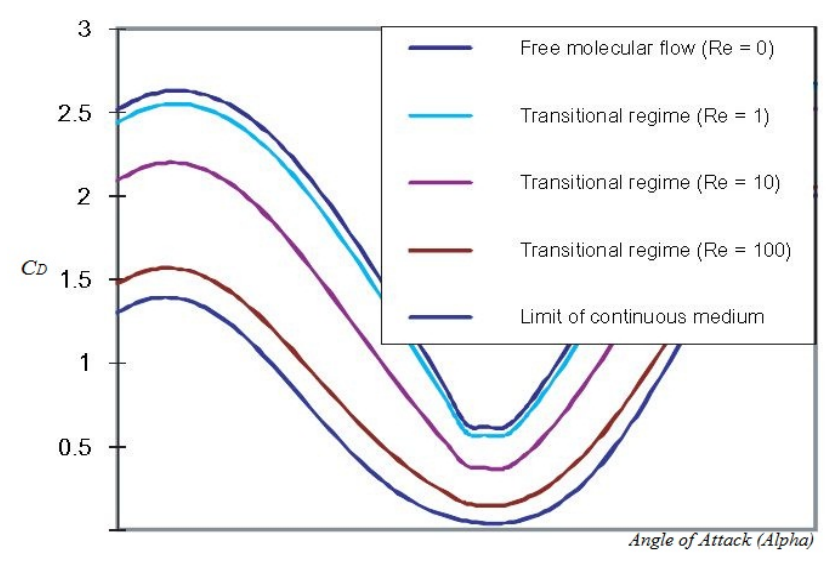

Figure 2. Drag coefficient for space shuttle

\section{Results and Discussion}

The calculation results of the coefficients of drag force $C_{x}$, lift force $C_{y}$ and pitching moments $m_{z}$ with value of the angle of attack $\alpha$ ranging from -90 to +90 degrees for the space vehicle "Clipper, design of TsAGI (fig. 3)" [18] are described.

The aerodynamic characteristics of vehicle $\left(C_{x}, C_{y}\right.$ and $\left.m_{z}\right)$ are calculated according to equations as below

$$
\begin{gathered}
C_{x}=\frac{F_{x}}{\frac{1}{2} \rho_{\infty} V_{\infty}^{2} S_{r e f}}, C_{y}=\frac{F_{y}}{\frac{1}{2} \rho_{\infty} V_{\infty}^{2} S_{r e f}}, \\
m_{Z}=\frac{M}{\frac{1}{2} \rho_{\infty} V_{\infty}^{2} L_{r e f} S_{r e f}}
\end{gathered}
$$

where, $L_{r e f}, S_{r e f}$ - references length and surface; $F_{x}, F_{y}-$ resultant forces acting on the vehicle and $M$ - moment, respectively.
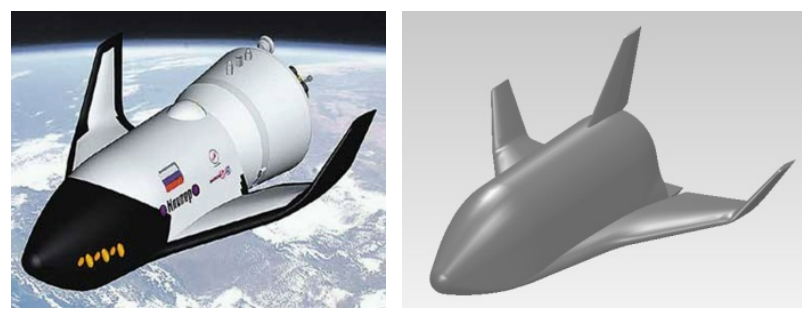

Figure 3. Geometrical representation of space vehicle "Clipper"

The calculation has been carried out through the method described in the previous section within the range of angles of attack $\alpha$ from -90 degree up to +90 degree with a step of 5 deg. The parameters of the problem are the following: specific heat ratio $\gamma=1.4$; temperature factor $t_{w}=T_{w} / T_{0}=0.001$; velocity ratio $=15$, Reynolds number $\mathrm{Re}_{0}=0,1,10,100,1000,10000$.

The dependencies $C_{x}(\alpha), C_{y}(\alpha), m_{z}(\alpha)$, at different values of Reynolds number are presented in Figs. 4-6. It is seen from these results that as the Reynolds number increases, the drag coefficient of the body diminishes (which can be explained by the decrease of normal and tangent stresses $p_{1}\left(\operatorname{Re}_{0}\right)$ and $\left.\tau_{1}\left(\operatorname{Re}_{0}\right)\right)$, and meanwhile the total character of the dependency $C_{x}(\alpha)$ is not changed. The dependency $C_{y}(\alpha)$ is asymmetric at $\operatorname{Re}_{0} \rightarrow 0$, so the value $C_{y}$ at negative angles of attack. Let us note that the balancing angle of attack of the vehicle at $\operatorname{Re}_{0} \rightarrow \infty$ is 0 . The values of $m_{z}$ are quite sensitive to the variation of $\mathrm{Re} 0$. At an increase of $\mathrm{Re}_{0}, m_{z}$ changes its sign at positive angles of attack. The value $\operatorname{Re}_{0} \sim 10$ is limited value. At $\operatorname{Re}_{0} \rightarrow \infty$, the peak value of $m_{z}=-0.03$ at positive angles of attack is reached at $\alpha \approx 40 \mathrm{deg}$. The vehicle is completely unstable in pitch at $\operatorname{Re}_{0} \rightarrow \infty$.

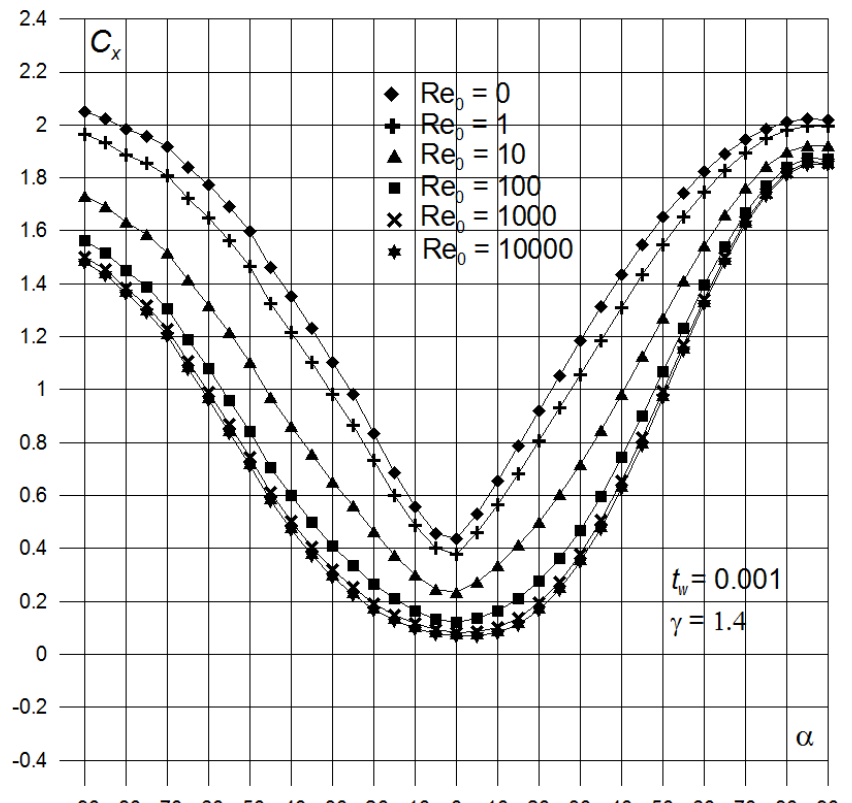

Figure 4. $C_{x}$ for space vehicle "Clipper"

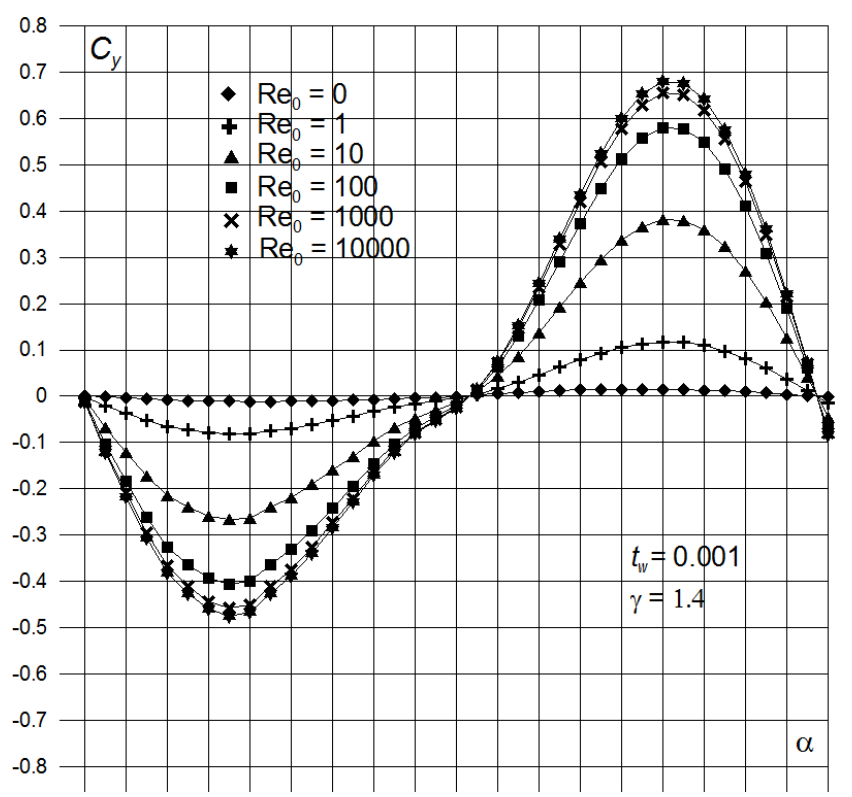

$\begin{array}{lllllllllllllllllll}-90 & -80 & -70 & -60 & -50 & -40 & -30 & -20 & -10 & 0 & 10 & 20 & 30 & 40 & 50 & 60 & 70 & 80 & 90\end{array}$

Figure 5. $C_{y}$ for space vehicle "Clipper" 


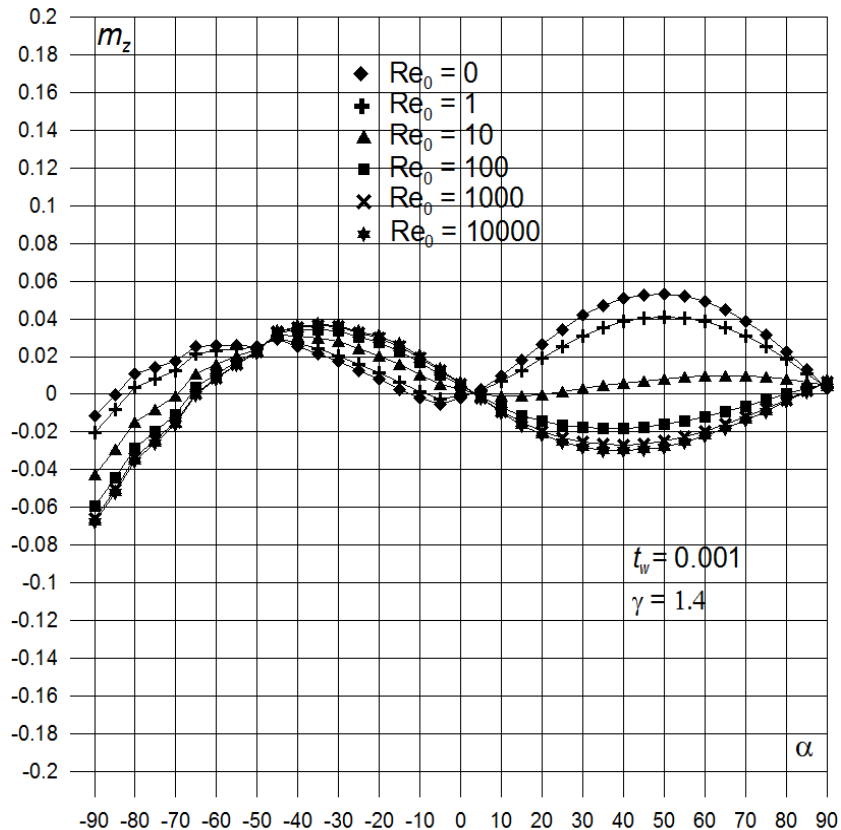

Figure 6. $m_{z}$ for space vehicle "Clipper"

\section{Conclusions}

The different approach to calculate aerodynamic characteristics of space vehicle in rarefied gas flow based on the approximate engineering method was carried out. The results of calculation of aerodynamic characteristics for new generation space vehicle by using engineering method with taking into account various Reynolds numbers are presented. Thus, the approximate engineering method gives good results for wide range of vehicle bodies in transitional regimes. The method and obtained results can be applied in the future space vehicle designs.

\section{Acknowledgements}

The reported study was partially supported by RFBR, research project No. 11-07-00300-a. Authors would like to thank RFBR for their support.

\section{REFERENCES}

[1] N.M. Kogan, "Rarefied Gas Dynamic," New York, Plenum, 1967.

[2] O.M. Belotserkovskii, Y.I. Khlopkov, "Monte Carlo Methods in Mechanics of Fluid and Gas," World Scientific Publishing Co. N-Y, London, Singapore, Beijing, Hong Kong, 2010, $268 \mathrm{p}$.

[3] J.N. Moss, C.G. Glass, B.R. Hollis and J.W. Van Norman, "Low Density Aerothermodynamics of the Inflatable Reentry Vehicle Experiment", AIAA Paper 2006-1189, 2006.
[4] T.J. Horvath, S.A. Berry, N.R. Merski, "Hypersonic Boundary/Shear Layer Transition for Blunt to Slender Configurations - A NASA Langley Experimental Perspective," Enhancement of NATO Military Flight Vehicle Performance, Czech Republic, 4-7 October 2004, RTO-MP-AVT-111

[5] M.S. Ivanov, "Statistical Simulation of Reentry capsule Aerodynamics in Hypersonic Near-Continuum Flows" RTO lecture series, Models and Computational Methods for Rarefied Flows (AVT-194), 2011. EN-AVT-194-18

[6] R.D. Neumann, "Missions and requirements. Special Course Aerothermodynamics of Hypersonic Vehicles," AGARD Report 761, Neuilly sur Seine, France, 1988.

[7] E.H. Hirschel, "Basics of Aerothermodynamics," Progress in Astronautics and Aeronautics, AIAA, Springer-Verlag, Berlin/Heidelberg/New York, 2005.

[8] G.A. Bird, "Molecular Gas Dynamics and the Direct Simulation of Gas Flows," Oxford University Press, 1994, $479 \mathrm{p}$.

[9] J.D. Anderson, "Hypersonic and High-Temperature Gas Dynamics," New York: McGraw-Hill, 1988, 702 p.

[10] L. Lees, "Hypersonic Flow," Processing of 5th International Aeronautical Conference, Los Angeles, New York, 1955, pp. 241-275.

[11] J.J. Bertin, R.M. Cummings, "Critical Hypersonic Aerothermodynamic Phenomena," Annual Review of Fluid Mechanics, Vol. 38, 2006, pp. 129-157. doi: 10.1146/annurev.fluid.38.050304.092041

[12] A.H. Shapiro, "The Dynamics and Thermodynamics of Compressible Fluid Flow,” Wiley, New York, 1953.

[13] D.E. Glass, M.S. Holden, A.R. Wieting, "Effect of Leading Edge Sweep on Shock/Shock Interference at Mach 8," 27th AIAA Aerospace Science Meeting, AIAA Paper 89-0271, Reno, NV, 1989.

[14] D.E. Reubush, M.E. Omar, "Pressure and Heat Transfer Investigation of a Modified NASP Baseline Configuration at $\mathrm{M}=6$," 27th AIAA Aerospace Science Meeting, AIAA Paper 89-0246, Reno, NV, 1989.

[15] K. Fujimoto, K. Fujii, N. Tsuboi, "CFD Prediction of the Aerodynamic Characteristics of Capsule-like Configurations for the Future SSTO Development," 41st AIAA Aerospace Science Meeting, AIAA Paper 03-0912, Reno, NV, 2003.

[16] Zay Yar Myo Myint, A.Yu. Khlopkov, "Aerodynamic Characteristics of an Aircraft with a Complex Shape Taking into Account the Potential of Molecular Flow Interaction with a Surface," TsAGI Science Journal, Vol. 41, No. 5, 2010, pp. 551-566. doi:10.1615/TsAGISciJ.v41.i5.50

[17] Yu.I. Khlopkov, Zay Yar Myo Myint, A.Yu. Khlopkov, "Aerodynamic Investigation for Prospective Aerospace Vehicle in the Transitional Regime," International Journal of Aeronautical and Space Sciences, KSAS, Korea, Vol. 14, No. 3, 2013, pp. 215-221. doi:10.5139/IJASS.2013.14.3.215

[18] A.V. Vaganov, S. Drozdov, A.P. Kosykh, G.G. Nersesov, I.F. Chelysheva, V.L. Yumashev, "Numerical Simulation of Aerodynamics of Winged Reentry Space Vehicle," TsAGI Science Journal, Vol. 40, No. 2, 2009, pp. 131-149. doi:10.1615/TsAGISciJ.v40.i2.10 